\title{
Live-Cell Mesothelioma Biobank to Explore Mechanisms of Tumor Progression
}

\author{
Kathrin Oehl', Jelena Kresoja-Rakic ${ }^{2}$, Isabelle Opitz ${ }^{2}$, Bart Vrugt ${ }^{1}$, Walter Weder ${ }^{2}$, \\ Rolf Stahel ${ }^{3}$, Peter Wild ${ }^{1}$ and Emanuela Felley-Bosco ${ }^{2 *}$
}

'Department of Pathology and Molecular Pathology, University Hospital Zürich, Zürich, Switzerland, ${ }^{2}$ Laboratory of Molecular Oncology, Division of Thoracic Surgery, University Hospital Zürich, Zürich, Switzerland, ${ }^{3}$ Cancer Center Zürich, University Hospital Zürich, Zürich, Switzerland

Experimental models closely representing in vivo conditions allow investigating mechanisms of resistance. Our aims were to establish a live-cell biobank of malignant pleural mesothelioma (MPM) samples and to obtain proof of principle that primary culture chemoresistant models, mimicking tumor progression observed in patients, can be obtained in vitro, providing a useful tool to investigate underlying mechanisms. Primary mesothelioma cultures were established from 235 samples between 2007 and 2014. Of

OPEN ACCESS

Edited by:

Marco Lucchi,

University of Pisa, Italy

Reviewed by:

Janaki Deepak,

University of Maryland,

Baltimore, United States

Anca Maria Cimpean,

University of Medicine and

Pharmacy, Timisoara, Romania

${ }^{*}$ Correspondence:

Emanuela Felley-Bosco emanuela.felley-bosco@usz.ch

Specialty section: This article was submitted to

Thoracic Oncology, a section of the journal

Frontiers in Oncology

Received: 13 October 2017 Accepted: 05 February 2018 Published: 23 February 2018

Citation:

Oehl K, Kresoja-Rakic J, Opitz I, Vrugt B, Weder W, Stahel R, Wild P and Felley-Bosco E (2018) Live-Cell

Mesothelioma Biobank to Explore Mechanisms of Tumor Progression.

Front. Oncol. 8:40.

doi: 10.3389/fonc.2018.00040 two MPM patients, primary cultures obtained at different time points: at initial diagnosis, after neoadjuvant treatment at surgery and/or after tumor recurrence, were deeply investigated. Cells and corresponding tumor tissue were characterized by mesothelial protein and gene expression analysis. In addition, primary cultures from chemo naive patients were exposed to increasing doses of cisplatin/pemetrexed during three months and compared with non-treated cells in a cytotoxicity assay, and by selected profiling of senescence markers. In vitro chemoresistance in the primary mesothelioma cell cultures was associated with increased Thy1 (CD90) expression. Thy1 expression in MPM samples was significantly associated with poor overall survival in the TCGA MPM cohort. Our results illustrate that the establishment of a large live-cell MPM biobank contributes to a better understanding of therapy resistance observed in vivo, which eventually may lead to a more logical approach for developing new treatment strategies.

Keywords: mesothelioma, primary culture, tumor progression, chemoresistance, genetic profiling, mutations, copy number, cisplatin and pemetrexed

\section{INTRODUCTION}

Malignant pleural mesothelioma (MPM) is an asbestos-related cancer arising from mesothelial cells of the pleura. The prognosis of MPM patients is poor [median survival of 12 months (1)] likely because in most cases diagnosis is made in advanced disease stages. A recent large-scale study based on pathological samples has comprehensively characterized most genetic alterations and distinct molecular profiles in MPM (2). However, there is still the need of experimental models to define cancer driver activity of a given genetic alteration, like it has been done with the $\mathrm{Nf2-(3-5) \text {and }}$ Bap1-deficient mice $(6,7)$. Experimental models may closely resemble in vivo conditions and allow for the testing of novel therapeutic approaches to understand their mechanism of action.

Moreover, experimental models allow for the investigation of resistance mechanisms. Patientderived xenografts in humanized NSG mice (https://www.jax.org/news-and-insights/jax-blog/2015/ 
april/the-next-big-thing-in-cancer-modeling-patient-derivedxenografts-in-humaniz) would represent the best overall model but currently are difficult to implement for routine purposes. In contrast, cell lines are an easy accessible disease model (8) that is still accepted for certain investigations, such as molecular mechanism of drug action. Primary mesothelioma cultures were shown as valid model for mechanisms of resistance to pemetrexed, which, in combination with platinum compounds, is a component of current MPM standard neoadjuvant treatment (9). According to Cellosaurus (http://web.expasy.org/cgi-bin/cellosaurus/search) there are 402 human mesothelioma cell lines available. Of these cell models, only 11 have a well-profiled genotype (https://portals.broadinstitute.org/ccle/home) and for 45, fingerprinting for identification is available. In addition, long-term passaging leads to genetic drift caused by genomic instability. A recent study (10) demonstrated an upregulation of glycolytic and oxidative phosphorylation in commercial lines compared to primary cultures. In addition, primary cells maintain expression of podoplanin, which is lost in commercial lines and they show activation of the type I interferon signaling pathway (10).

In this study, we tested the hypothesis whether molecular profiling of primary mesothelioma cell culture obtained from patients with MPM are helpful to understand the mechanisms of acquired resistance to cisplatin/pemetrexed treatment in vivo.

\section{MATERIALS AND METHODS}

\section{Tumor}

Tissue samples were obtained from patients treated for MPM at the Department of Medical Oncology and Department of Thoracic Surgery between January 2007 and December 2014. The study has been approved by the Zurich Cantonal Ethics Committee (reference numbers StV 24-2005 and 29-2009). Written informed consent was obtained from all patients. Tumor samples obtained from diagnostic biopsies prior to neoadjuvant chemotherapy and the corresponding resection specimens were processed immediately for total RNA extraction using Qiagen RNAeasy kit (Qiagen, Hilden, Germany). In addition, another part of the tumor samples was embedded in OCT and immediately frozen at $-80^{\circ} \mathrm{C}$. The remaining tumor samples were fixed in $4 \%$ buffered formaldehyde for paraffin embedding.

\section{Cell Culture and Senescence-Associated- $\beta$-Gal Activity}

Primary mesothelioma cell cultures were generated as previously described (11). In order to establish a model for chemoresistance, two primary cultures from chemotherapy-naive epithelioid MPM specimens were continually exposed to low doses of cisplatin and pemetrexed according to a previously published method of cisplatin-resistance in ovarian cancer cell lines (12). These two cultures originated from two MPM patients. The first primary culture was taken from a biopsy of a male 69-year-old patient (P95), who had an epithelioid MPM (Figures S1 and S2 in Supplementary Material) and was enrolled into the SAKK17/04 study (13). The patient, therefore, underwent extrapleural pneumonectomy after neoadjuvant chemotherapy but relapsed 15 months later. He died
27 months after the initial diagnosis. The second culture was established from a female, 50-year-old patient (P236) with papillary epithelioid tumor morphology (Figure S1 in Supplementary Material). The patient underwent partial pleurectomy (Figure S2 in Supplementary Material) but relapsed 19 months later and was operated again, followed by chemotherapy. Unfortunately, the patient died 3 years after the first surgery. In the two chemo-naive primary cultures, cells were treated with cisplatin at the initial dose of 3 and $1 \mathrm{nM}$ pemetrexed. The treatment was conducted in three cycles, including 4 days of treatment and 3 days of recovery time. The concentration was doubled and the procedure was repeated until the concentration of cisplatin and pemetrexed reached 48 and $16 \mathrm{nM}$, respectively. Chemoresistance induction was evaluated based on the cytotoxicity to a subsequent exposure to cisplatin and pemetrexed. Doubling time was estimated as previously described (14). Primary cells were authenticated by DNA fingerprinting of short tandem repeat loci (Microsynth, Switzerland). Senescence-associated- $\beta$-gal activity was determined as previously described (15).

\section{Relative Gene Expression}

To extract RNA, Qiagen RNeasy kit was used. cDNA was determined as previously described (15). Selected gene expression [primers are listed in Table S1 in Supplementary Material and (15-17) analysis using MIQE compliant protocols (18)] was conducted as previously described.

\section{Western Blotting}

Whole cellular protein extracts were prepared in $95^{\circ} \mathrm{C}$ Laemmli sample buffer and mechanically sheared by pressing few times through syringes $(26 \mathrm{G})$. Protein concentration was determined using a Pierce ${ }^{\mathrm{TM}} 660 \mathrm{~nm}$ Protein Assay (Thermo Scientific). A total of $5 \mu \mathrm{g}$ protein per extract was separated on denaturing $10-20 \%$ gradient SDS-PAGE gels. Proteins were transferred on PVDF transfer membranes $(0.45 \mu \mathrm{m}$, Perkin Elmer). Membranes were probed with the following primary antibodies: anticalretinin (Sigma, HPA007306), mouse anti-actin (\#69100) from MP Biomedicals, N-Cadherin (BD Biosciences, 610920), YAP (Cell Signaling 4912), Mesothelin (Rockland Inc. 200-301-A88), GATA4 (C-20) (Santa Cruz sc-1237), p62 (Progen GP62-C), LC3B (Cell Signaling 2775S), p53 (DO-1) (Santa Cruz sc-126), Thy1 (H-110) (Santa Cruz sc-9163), and $\gamma$-H2AX (Millipore 05-636). Membranes were then incubated with the secondary antibody rabbit anti-mouse IgG-HRP (A-5420) from Antell, and goat anti-rabbit IgG-HRP (\#7074) from Cell Signaling. The signals were detected by enhanced chemiluminescence (ECL ${ }^{\mathrm{TM}}$ Western Blotting Reagents, GE Healthcare) and detected on photosensitive film (Super RX Fuji x-Ray Film, Fujifilm). Proteins were quantitated with densitometry using Image J software (Version 1.42q, USA).

\section{Immunofluorescence}

Primary cells were grown on 12-mm glass coverslips in 24-well plates. Cells were fixed for $10 \mathrm{~min}$ with $4 \%$ paraformaldehyde and permeabilized with $0.05 \%$ saponin for $5 \mathrm{~min}$. The cells were then incubated over night at $4^{\circ} \mathrm{C}$ with Calretinin (N-term) (Sigma HPA007306), Podoplanin (D2-40) (Dako M3619), YAP1 
(Cell Signaling 4912), Ki67 (Abcam ab8191), Phalloidin 488 (molecular probes A12379), Thy1 (H-110) (Santa Cruz sc-9163), and N-Cadherin from Dako (M3613) diluted in PBS containing $1 \%$ bovine serum albumin. Secondary antibodies, Alexa Fluor 488-conjugated goat anti-rabbit IgG (Life Technologies A11034) and Alexa Fluor 555-conjugated goat anti-mouse IgG (Life Technologies A21424) antibody was added for $1 \mathrm{~h}$ at RT. Nuclear DNA was stained using DAPI. Coverslips were mounted using Prolong Gold antifade reagent (Life Technologies). Images were acquired using an Olympus BX61 microscope (Schwerzenbach, Switzerland) equipped with an F-view camera for conventional fluorescence imaging. The image capture was controlled with the AnalySISPro software (Soft Imaging System, Münster, Germany).

\section{Surface Phenotyping}

Cells resuspended in ice-cold PBS/2mM EDTA were incubated with antibodies against Thy $1(\mathrm{H}-110)$ (Santa Cruz sc-9163) at $4^{\circ} \mathrm{C}$ for $30 \mathrm{~min}$ in the dark. Secondary antibody (PE-labeled, Dianova) was incubated at $4^{\circ} \mathrm{C}$ for $30 \mathrm{~min}$ in the dark. After washing with PBS cells were resuspended in ice-cold PBS/2mM EDTA and fluorescence was measured on an Attune flow cytometer (Applied Biosystems) and analyzed with the Attune cytometric software v1.2.5 (Applied Biosystems).

\section{Genetic Alterations}

For DNA isolation from formalin-fixed, paraffin-embedded (FFPE) tissue, two punches of $0.6 \mathrm{~mm}$ were taken from the tumor of each FFPE Block. DNA was extracted using the Maxwell 16 FFPE Tissue LEV DNA Purification Kit (Promega) according to the manufacturer's protocol. Isolated DNA was diluted in nuclease-free water and the concentration was measured by fluorometric quantitation using the Qubit 2.0 fluorometer (Thermo Fisher Scientific).

For targeted amplicon-based sequencing, we developed a custom-designed Ion AmpliSeq MPM Panel (Thermo Fisher Scientific), covering the coding regions of 30 genes that were reported to be commonly mutated in MPM. $10 \mathrm{ng}$ of either FFPE tumor or primary cell DNA were used as an input for library preparation, following the Ion AmpliSeq DNA Library Preparation user guide (Thermo Fisher Scientific). Template preparation and sequencing was performed according to the manufacturer's protocol (Ion PI Hi-Q OT2 200 Kit User Guide and Ion PI Hi-Q Sequencing 200 Kit, Thermo Fisher Scientific). Alignment, variant calling, and filtering of the resulting data were performed with Ion Reporter 5.0 (Thermo Fisher Scientific). The filtering chain included removal of all synonymous mutations, variants that are found in the UCSC Common SNPs database, variants with an allele ratio below $10 \%$ or a read count below 50. Additionally, all mutations were visually inspected, using the Integrative Genomics Viewer Software (Broad Institute).

For array-based genome wide copy number analysis, OncoScan FFPE microarrays (Affymetrix) were conducted at IMGM Laboratories GmbH (Martinsried, Germany). An input amount of 79.2 ng DNA was used for the assay. Molecular inversion probe processing was carried out according to the OncoScan FFPE Assay Kit Protocol (Affymetrix). For data analysis, the
AGCC Viewer v4.2.1567 and the OncoScan Console v1.3.0.39 were used (Affymetrix). Copy number variation (CNV) and loss of heterozygosity $(\mathrm{LOH})$ analysis was performed using the Nexus Express for OncoScan v3.1 software. CNVs were visualized using Circos19. Oncoscan have been deposited in GEO (https://www. ncbi.nlm.nih.gov/geo/query/acc.cgi?acc=GSE109305).

\section{Statistics}

Data are expressed as mean \pm SD. Statistical analysis was performed using Mann-Whitney $U$ tests using StatView 5.0.1 (SAS institute) and $t$-test using GraphPad. Differences were considered statistically significant at $p<0.05$.

\section{RESULTS}

\section{Primary Cultures Reflect Tumor Characteristics}

Between 2007 and 2014, 235 surgical samples were obtained and processed, as described in Figure 1A. After preservation of tumor material for histopathological evaluation, remaining parts of the tumor were processed in order to extract RNA. Derived cDNA was then used for quality control assays, i.e., for calculation of the MPM score (15), allowing to determine that $82 \%$ of the samples contained at least $50 \%$ tumor cells. In case of large representative tumor tissue, additional parts of the fresh sample were used to establish primary cultures, either in the presence of serum (11) or in the absence of serum (17), for enzymatic analysis (15) or in situ hybridization (17). Successful primary cultures from "bona fide" tumor samples, defined as sufficiently growing cells for collecting RNA and total protein lysates, and preparing frozen stocks, was achieved in $68 \%$ of initial samples (159/235).

Primary cultures from two patients (P236 and P95) for whom samples were available at different stages of the disease (for nomenclature defining the different samples from these patients see Figure S2 in Supplementary Material) and where we had observed maintenance of epithelioid histology in one (P236) and epithelial to mesenchymal transition (EMT) in the second (P95), were selected for this proof of principle study. It is worth noting that EMT was evaluated on surgical specimens excluding any diagnostic bias introduced by single biopsy analysis. For these two patients, established primary cultures from different time points were compared with matching initial tumor samples by short tandem repeat analysis (Table S2 in Supplementary Material), Oncoscan CNV testing, and amplicon panel sequencing (Figure 1B). While few genetic alterations were present in P236, consistent with current knowledge on papillary mesothelioma (19), P95 was characterized by a widespread LOH including NF2, BAP1, and TP53 tumor suppressor genes, as well an additional truncating mutation in NF2 (c.553G > T/p. Glu185Ter, no Cosmic annotation) and a point mutation in TP53 (c.839G > C/p.p.Arg280Thr, cosm254987).

$\mathrm{N}$-cadherin, mesothelin, and calretinin MPM biomarker mRNA and protein expression was determined (Figure 1C) and decreasing calretinin expression reflected the evolution of the tumor. A high level of YAP expression was observed in all cultures. Culture heterogeneity was evidenced in one of the 
patient (P236_cells) by immunofluorescence analysis, revealing that not all cells stained for podoplanin (D2-40), Ki-67, nuclear YAP, or nuclear calretinin, while all cells expressed $\mathrm{N}$-cadherin (Figure 1D). Changes in mesothelin, calretinin and podoplanin gene expression levels were also evaluated in tumor samples (Figure 1E). Differences observed in vivo for selected
mRNAs involved either in differentiation (HES1, RUNX2), EMT (GREM1, CTGF), senescence (PUMA, LOX, PAI1), drug transport (SLC22A4, ABCC1), cell cycle (CALB2 (20), CCND1, $C D K N 2 A)$, response to oxidative stress or hypoxia (HO-1, CAIX, GLUT1), or MPM-associated surface proteins (PDPN, THY1, CD276, TNFRSF10B) were weakly $\left(R^{2}=0.2641\right)$ but significantly
A

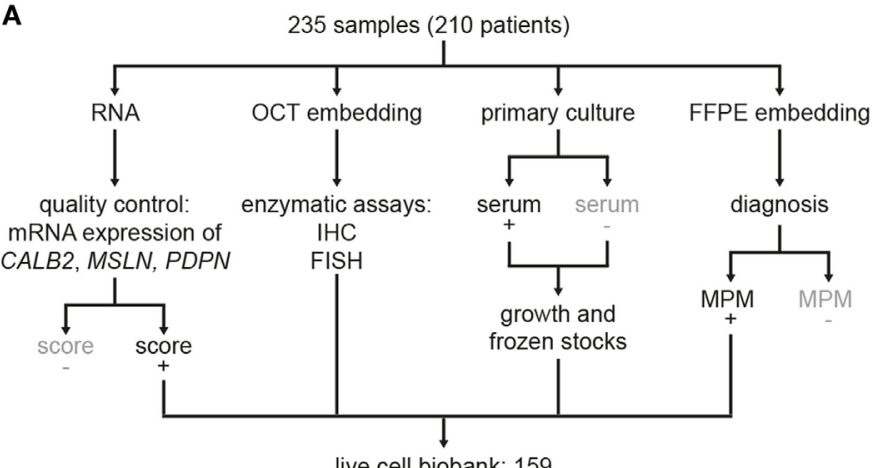

C
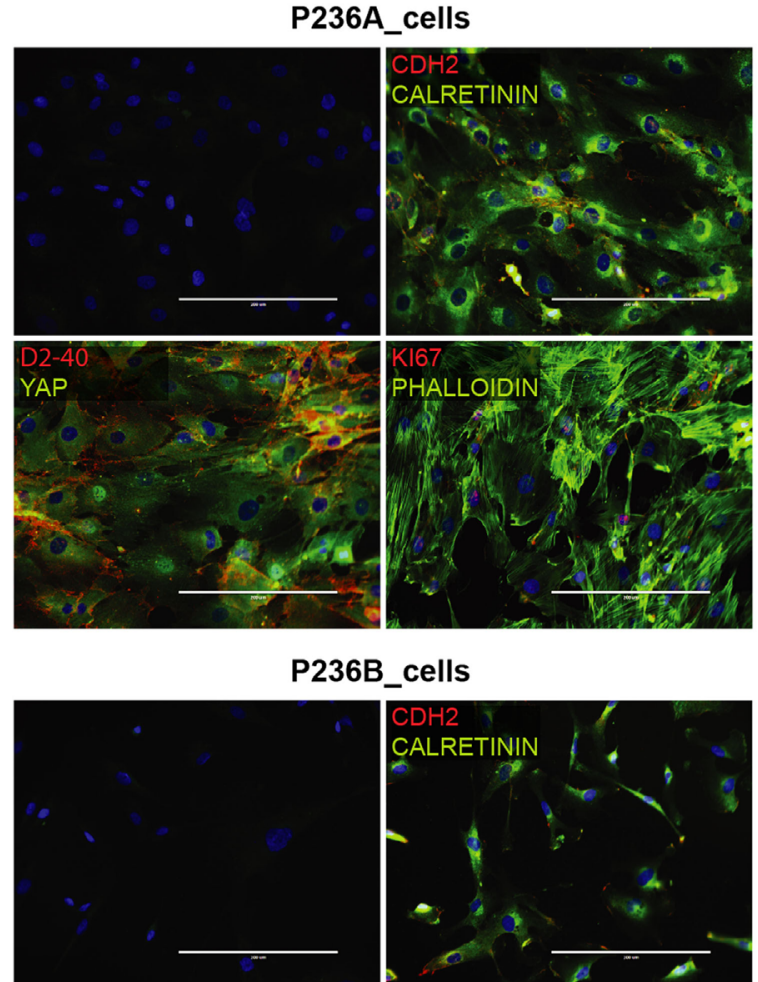

\section{cells}
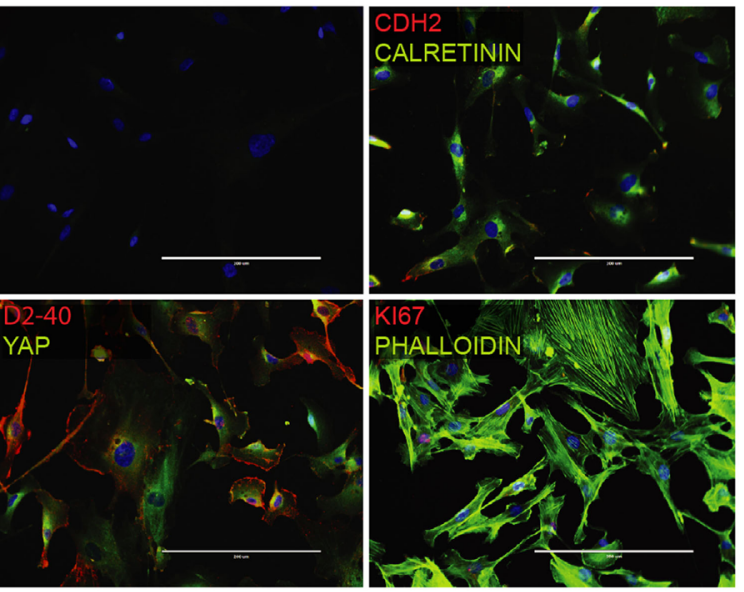

B

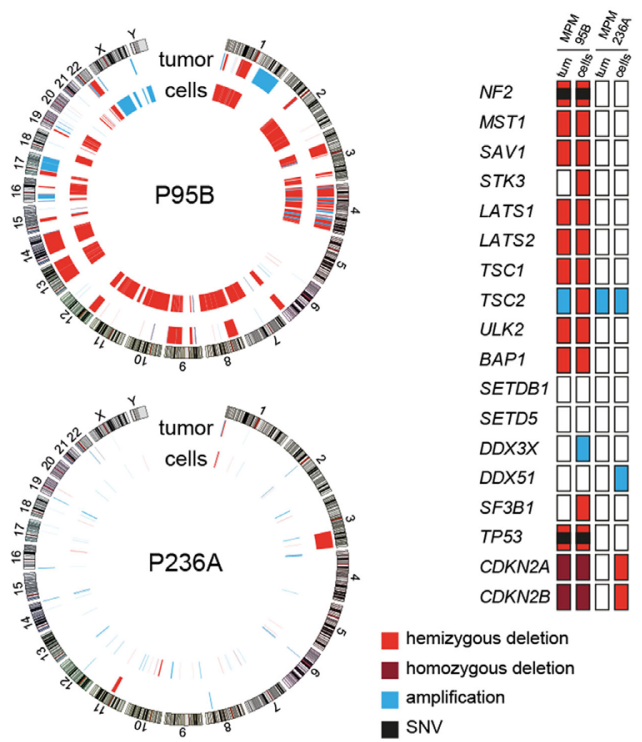

D
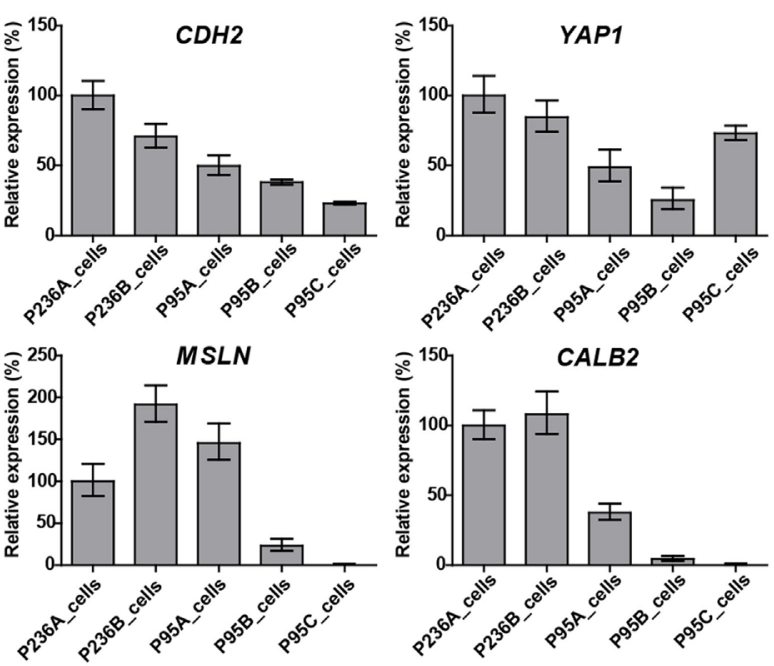

FIGURE 1 | Continued

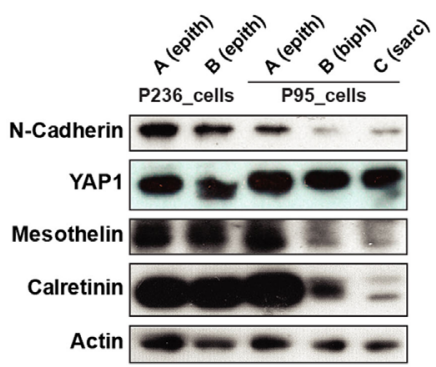


E

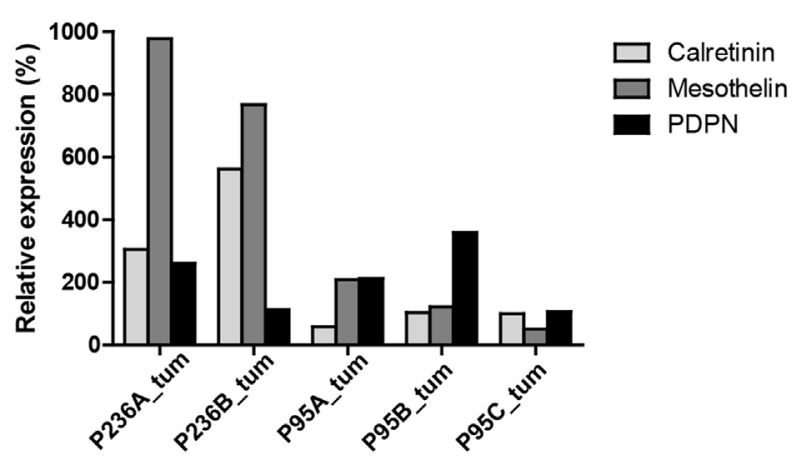

$\mathbf{F}$

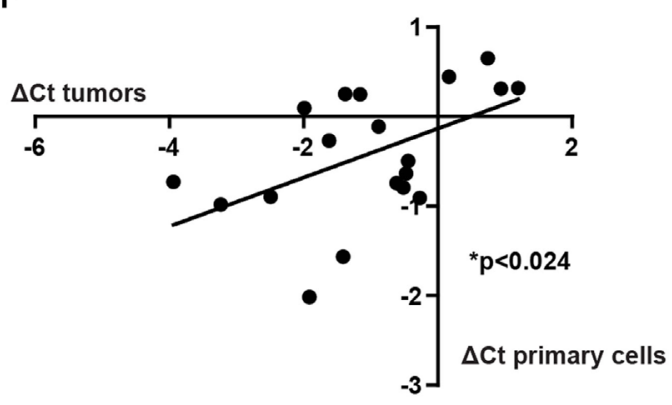

FIGURE 1 | (A) Tumor processing flow-chart of 235 samples, which were processed for RNA extraction, cell culture and embedding either in PFA or OCT. Samples showing attributes which are grayed in the chart, were not used in the live cell biobank. (B) Circos whole genome copy number variations (CNVs) view of tumor and primary cell culture in patients malignant pleural mesothelioma (MPM) 236 and MPM95. The quilt plot highlights the CNV and SNVs in genes that are part of MPM landscape (2). (C) Immunofluorescence analysis of selected markers in primary culture from patient MPM236. Scale bar 200 $\mu$ m. (D) Selected genes expression analysis at mRNA (upper panel) and protein (lower panel) level in primary cultures derived from samples from two patients, MPM236 and MPM95. The latter one underwent EMT during disease progression. (E) Selected genes expression analysis at mRNA in tumor samples from patients MPM236 and MPM95. (F) Significant correlation between gene expression changes in tumor and primary culture from patient MPM236 at passage 3.
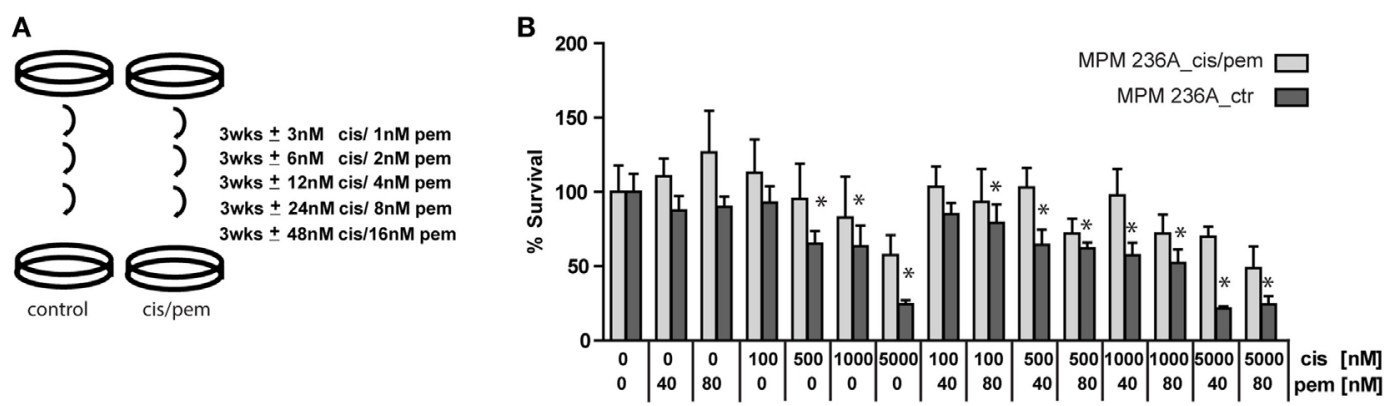

FIGURE 2 | (A) Establishment in vitro of chemoresistant cell lines by exposure to gradually increasing doses of cisplatin/pemetrexed. (B) Cis/pem line established from patient P236A_cells is chemoresistant compared to control when challenged with pemetrexed/cisplatin. Two-way ANOVA with Bonferroni's post hoc test, ${ }^{\star} p<0.01$.

correlated with differences observed in primary cultures in patient P236 (Figure 1F). This supports the idea of using primary cultures for the functional investigation of mechanisms underlying tumor evolution.

\section{Development of Chemoresistance Is Accompanied by Increased Levels of DNA Damage, Autophagy and Senescence Marker $\boldsymbol{\beta}$-Galactosidase}

Malignant pleural mesothelioma chemoresistance models have been described after $96 \mathrm{~h}$ exposure of human mesothelioma cell lines to high doses of pemetrexed (9) or after 15 cycles of combination of treatment with IC50 concentrations of cisplatin plus pemetrexed during $72 \mathrm{~h}$ followed by a recovery time in a rat cell line (21). We opted for a method that has been used in a study inducing cisplatin-resistance to ovarian cancer cell lines (12) (Figure 2A) to avoid acute induction of senescence-associated growth arrest observed using high doses of pemetrexed (9). Using this approach, we successfully generated a chemoresistant model for primary cells derived from patient P236 (Figure 2B) but not for primary cells derived from patient P95 (Figure S3 in Supplementary Material). This was accompanied by a higher basal chemoresistance in primary cells derived from patient P95 (IC50 2.5 vs. $1.5 \mu \mathrm{M}$ in the primary culture from patient P236). Doubling time in the cultures exposed or not to cisplatin/pemetrexed did not differ in either of the two patients (Figure S4 in Supplementary Material) excluding that growth kinetics were the underlying mechanism of resistance.

We have previously described the occurrence of senescence in MPM patients treated with chemotherapy (15). Because senescence is a stress response involving multiple effector mechanisms, including DNA damage response $(22,23)$, senescence-associated secretion phenotype (SASP) (24-28) and autophagy (29), we analyzed markers involved in these pathway. Consistent with development of chemoresistance in the cisplatin/pemetrexed primary culture from patient P236, we observed increased levels of senescence-associated $\beta$-gal activity (Figure 3A), autophagy markers LC3B-II (53\% increase) and p62 (41\% increase) and appearance of SASP-associated (28) GATA-4 (Figure 3B), 


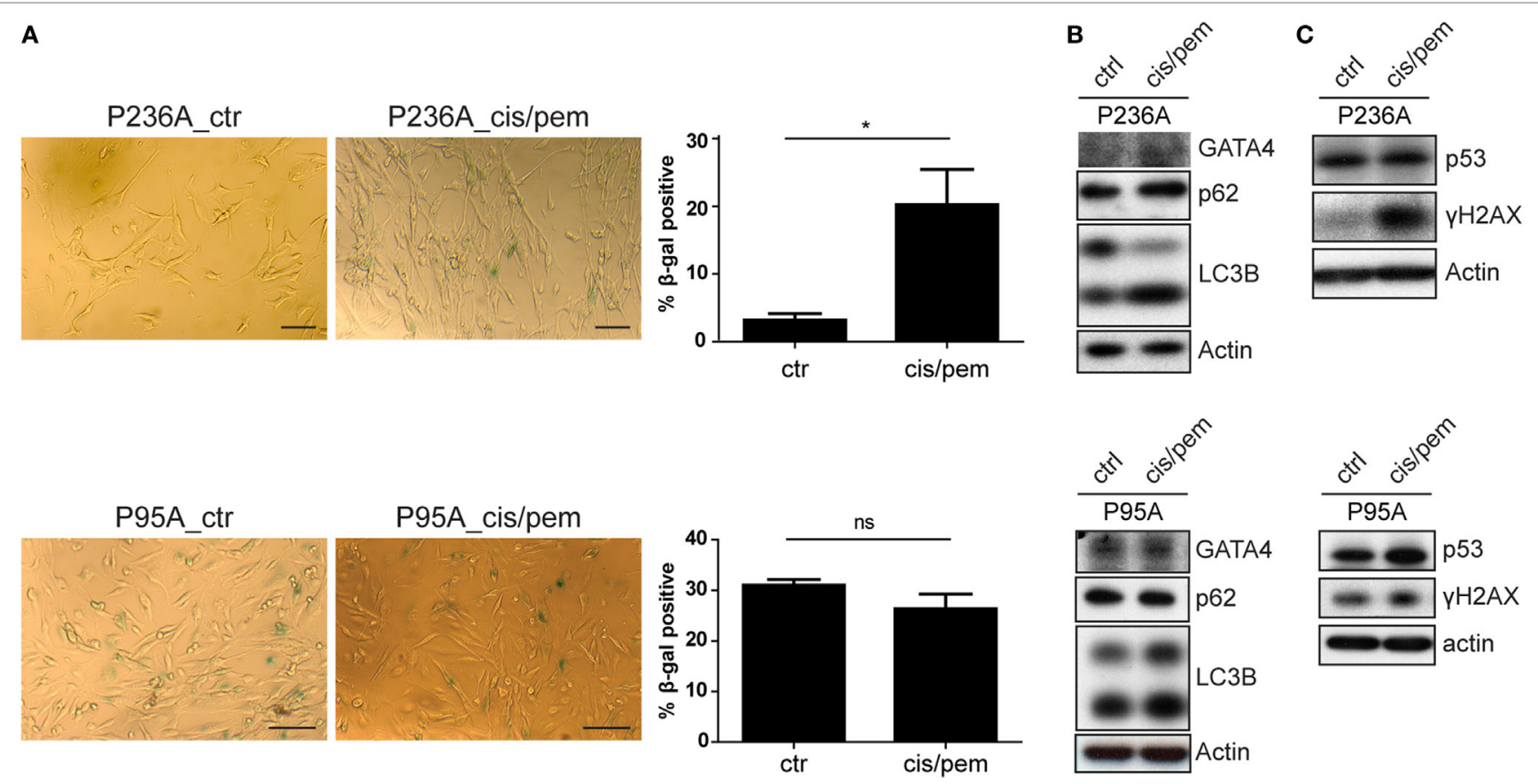

FIGURE 3 | (A) Senescence-associated beta-galactosidase staining in control and cis/pem adapted primary cultures from patient malignant pleural mesothelioma (MPM) 236 and MPM95. Scale bar 25 m. (B) Expression of senescence-associated secretion phenotype-associated GATA-4 and autophagy activation LC3B and p62. (C) DNA-damage response markers $\gamma$-H2AX and p53 stabilization.

and $\mathrm{H} 2 \mathrm{AX}$ phosphorylation (more than sevenfold increase, Figure 3C). Importantly, basal levels of senescence-associated $\beta$-gal activity were higher in patient P95, and did not differ in cisplatin/pemetrexed treated cultures. In the same patient the expression of GATA- 4 was already detected in the control primary culture and did not increase further in the cisplatin/pemetrexed culture. Increased levels of the other markers in the cisplatin/ pemetrexed compared to control culture were smaller compared to changes observed in patient P236. All in all, this data confirms that chemotherapy can induce resistance via induction of senescence but also indicates that the cancer genetic background per se is likely responsible for senescence and resistance due to genomic instability.

\section{Increase in Thy 1 Expression in Acquired-Chemoresistance Line}

In order to get more insight into events occurring during the development of chemoresistance in primary cultures from patient P236 exposed to cisplatin/pemetrexed, we analyzed the mRNA expression of autophagy/senescence-associated $I L-6$ and PAI- $1(15,30)$ and chemoresistance-development associated Thy1 (31) at three different time points during culture treatment (Figure 4A) and in the original primary cultures. Consistent with increased level of SASP-associated GATA4, we observed upregulation of $I L-6$ at the first two time points and in the primary culture obtained in the progressing tumor. PAI-1 expression followed a similar pattern. THY1 was decreased at the first time point then it increased, as also observed in the progressing tumor. We had identified Thyl
$\mathrm{N}$-glycoprotein on the cell surface of mesothelioma cells in MPM tumors (32), therefore, we analyzed Thyl positive cells by immunofluorescence in both, control and cisplatin/pemetrexed adapted primary cultures and original primary cultures from the two specimens obtained from the tumor. We observed that Thy was expressed at increased levels in primary cultures obtained during cisplatin/pemetrexed adaptation (Figure 4B, upper panel) and as well during tumor progression (Figure 4B, lower panel), although to a lower extent. For quantification, we performed flow cytometric analyses (Figure 4C) and observed a 10-fold increase of expression of Thy1 surface protein in cisplatin/pemetrexed exposed primary cells at the third time point, confirming our immunofluorescence data. In addition, we detected Thyl protein expression by WB (data not shown) in cisplatin/pemetrexed adapted cells. The observation associating higher levels of Thy1 with tumoral cell progression is consistent with Thy1 high expression levels with worst overall survival in TCGA (Figure S5 in Supplementary Material).

In the process of trying to better understand mechanisms regulating Thyl expression, we realized that we had used primers (15) based on UCSC Genome Browser on Human Feb. 2009 Assembly, which detect both THY1 and long non-coding RNA (ncRNA) RP11-334E6.12, a lncRNA in antisense orientation compared to THY1 and which partially overlaps with THY1 3'UTR. We, therefore, redesigned primers recognizing individually THY1 and lncRNA RP11-334E6.12, and observed a strong correlation between lncRNA RP11-334E6.12 and THY1 expression (Figure 4D), which is consistent with correlated expression between these two gene expression reported in G.Tex (33) (Figure 

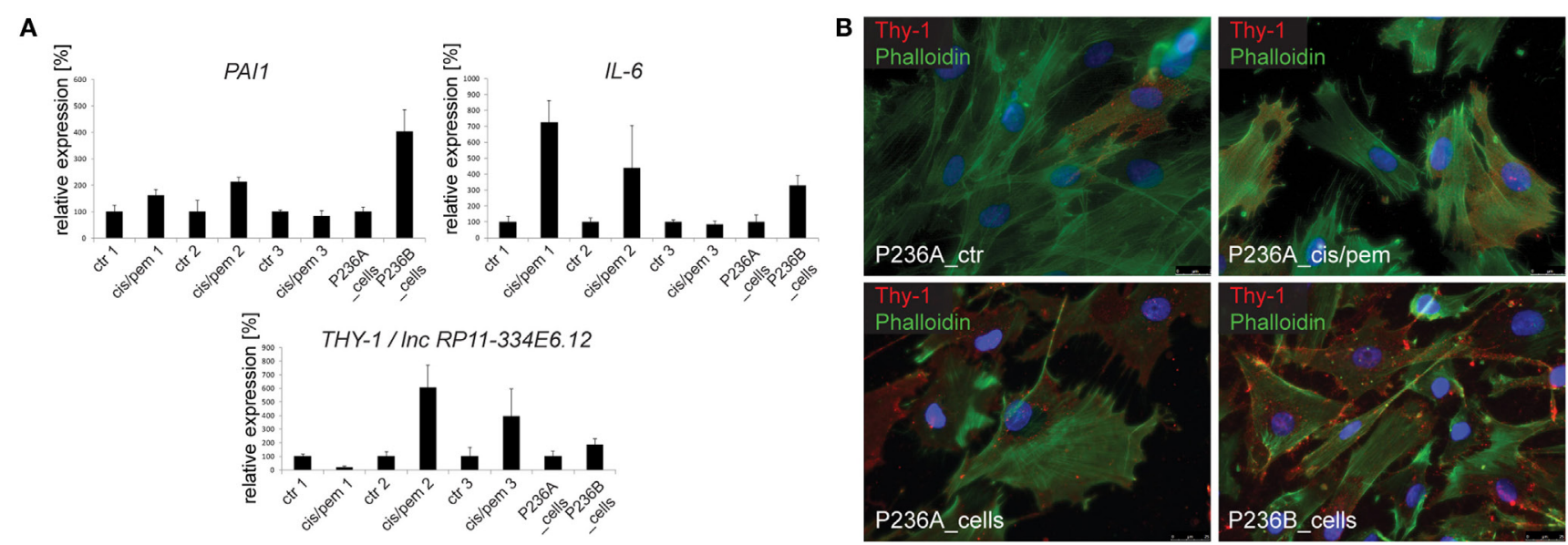

C
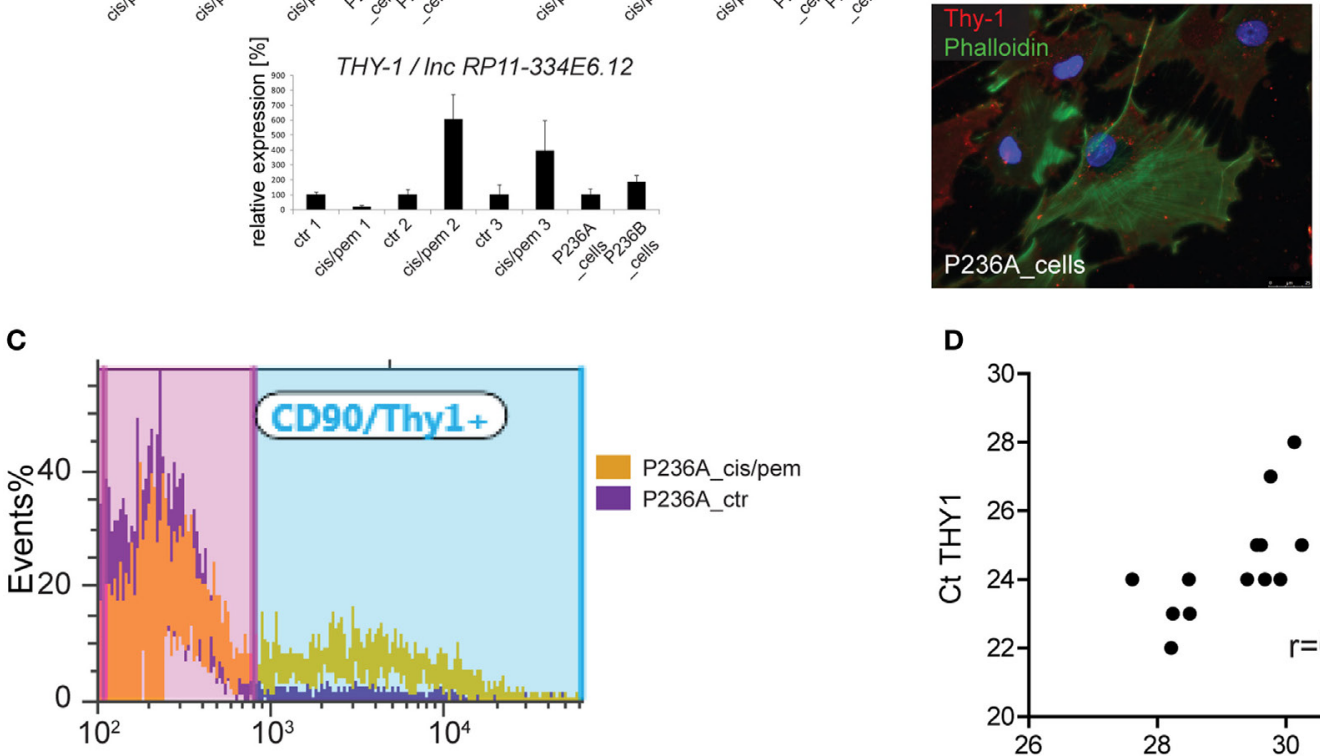

D

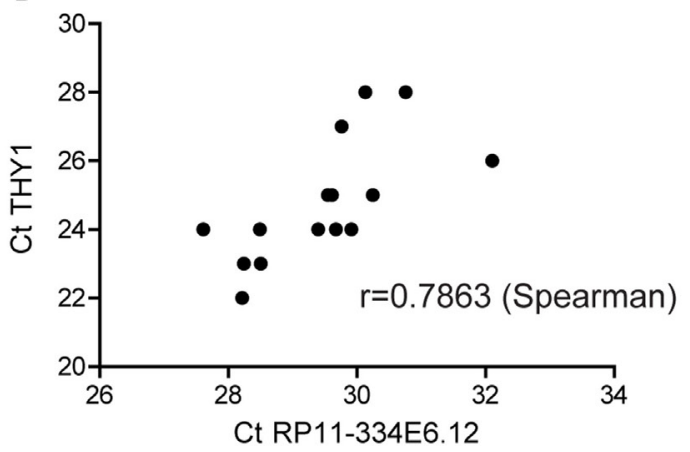

FIGURE 4 | (A) Expression of senescence-associated genes IL-6 and PAI-1 and THY1/nnc RP11-334E6.12 during chemoresistance development or tumor progression. (B) Immunofluorescence analysis of Thy1 expression cis/pem and control lines and original primary cultures from tumor samples of malignant pleural mesothelioma (MPM) 236 during tumor progression. (C) Flow cytometry analysis of Thy1 expression in cis/pem vs. control line. (D) Inc RP11-334E6.12 mRNA expression correlates with THY1 expression in MPM tumors.

S6 in Supplementary Material). Taken together, this data indicate an enrichment of THY1 positive cells during chemoresistance development and a possible role for lnc RP11-334E6.12 in such an increase.

\section{DISCUSSION}

A national effort in UK has resulted in the establishment of 26 primary mesothelioma cultures within the UK Mesobank (34). In this study, we tested the usefulness of an even larger collection of primary mesothelioma cell cultures, in some cases obtained from the same patient at different stages during disease progression. We aimed to carry out functional studies and provide evidence that changes observed in vitro and in vivo are similar.

Primary cultures in cancer research are of high value. Indeed, they represent primary intermediate before growth as xenograft, which, although providing a three-dimensional tissue environment have high-cost and low-throughput capacity. We have highlighted (17) the importance of primary culture and their growing conditions for maintenance of stem cell signaling pathways active in vivo $(2,35)$. In addition, they may represent the unique resource available for the $60 \%$ of MPM that do not grow as xenograft (36).
Although high-quality repositories such as biobanks and biomolecular resource infrastructures are being established (37), access to a large numbers of MPM biospecimens of high quality remains difficult. In our study, we deeply characterized samples from two patients, exemplifying the heterogeneity that can be found in real life, with one patient showing high levels of chromosomal instability while in the second only minor alterations were observed.

In the primary culture from the patient with abundant chromosomal instability (P95), a basal level of chemoresistance associated with senescence-associated phenotype was observed. This is consistent with the presence of the gain of function p53 mutation observed in this patient. Indeed p53Arg280Threo, although not fully oncogenic, has been shown to increase growth in soft agar of p53-deficient cells (38). TP53 is rarely mutated in MPM and in a recent high-through put study (2) mutations in this gene were absent in epithelioid MPM. However, datamining the catalog of somatic mutations in cancer (COSMIC, http:// www.sanger.ac.uk/cosmic), we found that TP53 mutations are described in 2/24 (8\%) epithelioid mesothelioma. For this patient, we have already described that the side population was enriched in tumor initiating properties in the primary culture established at the time of surgery, where tumor has evolved from epithelial to 
biphasic histotype (35), and Hedgehog signaling is maintained in primary cultures in serum-free conditions (17). Because we now show that a resistant phenotype against cisplatin/pemetrexed is present before chemotherapy, we infer that intrinsic properties of the tumor, including gain of function of TP53, deletion of NF2, and $C D K N 2 A$ participate to the evolution of resistant cells toward EMT.

The tumor from the second patient (P236) was of epithelioid morphology, maintaining the same histotype throughout the course of the disease and displayed absence of gross genetic abnormalities. In this context, it is worth noting that in triple negative breast cancer, $26 \%$ of cancers lack gross genetic abnormalities and this is likely because they originate from an epithelial precursor with stemness properties (39). The second patient was operated again after relapsing and received chemotherapy after the second surgery but relapsed again.

Primary mesothelioma cultures obtained at a 4-year interval have already been shown to demonstrate increased drug resistance but underlying mechanisms have not been examined yet (40). While investigating mechanisms underlying the development of chemoresistance, we observed increased Thy1 (CD90) expression. Thyl is GPI-anchored cell surface protein and in human the highest expression is observed in fetal thymic cells and subgroup of fibroblasts (41). Thy1 overexpression suppresses cell proliferation in ovarian and nasopharyngeal cancer (42-46), however, Thy1 expression in cancer stem-cells has mostly been associated with chemoresistance and increased tumor-initiating properties (47-49). Thyl expression has been also observed in four tumorigenic MPM primary cell cultures grown in low serum conditions (50). We show here that increased Thyl was associated with increased expression of lncRNA RP11-334E6.12. There is not much information about this lncRNA. A significant correlation between expression of IncRNA RP11-334E6.12 and developmental processes has been documented in esophageal cancer (51); overexpression has been documented in triple negative breast cancer (52). Because this lncRNA is in antisense orientation compared to THY1, and partially overlaps with

\section{REFERENCES}

1. van Meerbeeck JP, Scherpereel A, Surmont VF, Baas P. Malignant pleural mesothelioma: the standard of care and challenges for future management. Crit Rev Oncol Hematol (2011) 78:92-111. doi:10.1016/j.critrevonc.2010.04.004

2. Bueno R, Stawiski EW, Goldstein LD, Durinck S, De Rienzo A, Modrusan Z, et al. Comprehensive genomic analysis of malignant pleural mesothelioma identifies recurrent mutations, gene fusions and splicing alterations. Nat Genet (2016) 48:407-16. doi:10.1038/ng.3520

3. Fleury-Feith J, Lecomte C, Renier A, Matrat M, Kheuang L, Abramowski V, et al. Hemizygosity of Nf2 is associated with increased susceptibility to asbestosinduced peritoneal tumours. Oncogene (2003) 22:3799-805. doi:10.1038/ sj.onc. 1206593

4. Altomare DA, Vaslet CA, Skele KL, De Rienzo A, Devarajan K, Jhanwar SC, et al. A mouse model recapitulating molecular features of human mesothelioma. Cancer Res (2005) 65:8090-5. doi:10.1158/0008-5472.CAN-05-2312

5. Jongsma J, Van Montfort E, Vooijs M, Zevenhoven J, Krimpenfort P, Van Der Valk M, et al. A conditional mouse model for malignant mesothelioma. Cancer Cell (2008) 13:261-71. doi:10.1016/j.ccr.2008.01.030

6. Xu J, Kadariya Y, Cheung M, Pei J, Talarchek J, Sementino E, et al. Germline mutation of Bap1 accelerates development of asbestos-induced malignant
THY1 3'UTR, it is tempting to speculate that it may participate in regulating THY1 expression. Although beyond the scope of this study, this hypothesis requires further investigation. In future studies, it would be of interest to investigate whether Thy1 expression contributes to chemoresistance and whether all MPMs are derived from a yet to be identified precursor cell.

\section{ETHICS STATEMENT}

Zurich Cantonal Ethics Committee (reference numbers StV 24-2005 and 29-2009).

\section{AUTHOR CONTRIBUTIONS}

EF-B and PW designed experiments. KO, JK-R, and EF-B carried out experiments and interpreted data. $\mathrm{KO}, \mathrm{JK}-\mathrm{R}$, and EF-B generated figures. $\mathrm{KO}$ and EF-B wrote the manuscript. All authors read and approved the final manuscript.

\section{ACKNOWLEDGMENTS}

We are grateful to Gabriela Ziltener and Manuel Ronner for skillful assistance.

\section{FUNDING}

This work was supported by the Swiss National Science Foundation Sinergia grant CRSII3 147697, the Stiftung für Angewandte Krebsforschung and Zürich Krebsliga. Funding bodies had no role in the design of the study and collection, analysis, and interpretation of data and in writing the manuscript.

\section{SUPPLEMENTARY MATERIAL}

The Supplementary Material for this article can be found online at http://www.frontiersin.org/articles/10.3389/fonc.2018.00040/ full\#supplementary-material. mesothelioma. Cancer Res (2014) 74:4388-97. doi:10.1158/0008-5472.CAN14-1328

7. Kadariya Y, Cheung M, Xu J, Pei J, Sementino E, Menges CW, et al. Bap1 is a bona fide tumor suppressor: genetic evidence from mouse models carrying heterozygous germline Bap1 mutations. Cancer Res (2016) 76:2836-44. doi:10.1158/0008-5472.CAN-15-3371

8. Wilding JL, Bodmer WF. Cancer cell lines for drug discovery and development. Cancer Res (2014) 74:2377-84. doi:10.1158/0008-5472.CAN-13-2971

9. Canino C, Mori F, Cambria A, Diamantini A, Germoni S, Alessandrini G, et al. SASP mediates chemoresistance and tumor-initiating-activity of mesothelioma cells. Oncogene (2012) 31:3148-63. doi:10.1038/onc.2011.485

10. Chernova T, Sun XM, Powley IR, Galavotti S, Grosso S, Murphy FA, et al. Molecular profiling reveals primary mesothelioma cell lines recapitulate human disease. Cell Death Differ (2016) 23:1152-64. doi:10.1038/cdd.2015.165

11. Thurneysen C, Opitz I, Kurtz S, Weder W, Stahel RA, Felley-Bosco E. Functional inactivation of NF2/merlin in human mesothelioma. Lung Cancer (2009) 64:140-7. doi:10.1016/j.lungcan.2008.08.014

12. Louie KG, Behrens BC, Kinsella TJ, Hamilton TC, Grotzinger KR, Mckoy WM, et al. Radiation survival parameters of antineoplastic drug-sensitive andresistant human ovarian cancer cell lines and their modification by buthionine sulfoximine. Cancer Res (1985) 45:2110-5. 
13. Stahel RA, Riesterer O, Xyrafas A, Opitz I, Beyeler M, Ochsenbein A, et al. Neoadjuvant chemotherapy and extrapleural pneumonectomy of malignant pleural mesothelioma with or without hemithoracic radiotherapy (SAKK 17/04): a randomised, international, multicentre phase 2 trial. Lancet Oncol (2015) 16:1651-8. doi:10.1016/S1470-2045(15)00208-9

14. Felley-Bosco E, Weston A, Cawley HM, Bennett WP, Harris CC. Functional studies of a germ-line polymorphism at codon 47 within the p53 gene. Am J Hum Genet (1993) 53:752-9.

15. Sidi R, Pasello G, Opitz I, Soltermann A, Tutic M, Rehrauer H, et al. Induction of senescence markers after neo-adjuvant chemotherapy of malignant pleural mesothelioma and association with clinical outcome: an exploratory analysis. Eur J Cancer (2011) 47:326-32. doi:10.1016/j.ejca.2010.09.044

16. Andre M, Felley-Bosco E. Heme oxygenase-1 induction by endogenous nitric oxide: influence of intracellular glutathione. FEBS Lett (2003) 546:223-7. doi:10.1016/S0014-5793(03)00576-3

17. Shi Y, Moura U, Opitz I, Soltermann A, Rehrauer H, Thies S, et al. Role of hedgehog signaling in malignant pleural mesothelioma. Clin Cancer Res (2012) 18:4646-56. doi:10.1158/1078-0432.CCR-12-0599

18. Bustin SA, Benes V, Garson JA, Hellemans J, Huggett J, Kubista M, et al. The MIQE guidelines: minimum information for publication of quantitative real-time PCR experiments. Clin Chem (2009) 55:611-22. doi:10.1373/ clinchem.2008.112797

19. Churg A, Allen T, Borczuk AC, Cagle PT, Galateau-Salle F, Hwang H, et al. Well-differentiated papillary mesothelioma with invasive foci. Am J Surg Pathol (2014) 38:990-8. doi:10.1097/PAS.0000000000000200

20. Kresoja-RakicJ,KapaklikayaE,ZiltenerG,DalcherD,SantoroR,ChristensenBC, et al. Identification of cis- and trans-acting elements regulating calretinin expression in mesothelioma cells. Oncotarget (2016) 7(16):21272-86. doi:10.18632/ oncotarget.7114

21. Hudson AL, Weir C, Moon E, Harvie R, Klebe S, Clarke SJ, et al. Establishing a panel of chemo-resistant mesothelioma models for investigating chemo-resistance and identifying new treatments for mesothelioma. Sci Rep (2014) 4:6152. doi:10.1038/srep06152

22. Bartkova J, Rezaei N, Liontos M, Karakaidos P, Kletsas D, Issaeva N, et al. Oncogene-induced senescence is part of the tumorigenesis barrier imposed by DNA damage checkpoints. Nature (2006) 444:633-7. doi:10.1038/ nature 05268

23. Di Micco R, Fumagalli M, Cicalese A, Piccinin S, Gasparini P, Luise C, et al. Oncogene-induced senescence is a DNA damage response triggered by DNA hyper-replication. Nature (2006) 444:638-42. doi:10.1038/nature05327

24. Kortlever RM, Higgins PJ, Bernards R. Plasminogen activator inhibitor-1 is a critical downstream target of p53 in the induction of replicative senescence. Nat Cell Biol (2006) 8:877-84. doi:10.1038/ncb1448

25. Kuilman T, Michaloglou C, Vredeveld LC, Douma S, Van Doorn R, Desmet CJ, et al. Oncogene-induced senescence relayed by an interleukin-dependent inflammatory network. Cell (2008) 133:1019-31. doi:10.1016/j.cell.2008.03.039

26. Wajapeyee N, Serra RW, Zhu X, Mahalingam M, Green MR. Oncogenic BRAF induces senescence and apoptosis through pathways mediated by the secreted protein IGFBP7. Cell (2008) 132:363-74. doi:10.1016/j.cell.2007.12.032

27. Rodier F, Coppe JP, Patil CK, Hoeijmakers WA, Munoz DP, Raza SR, et al. Persistent DNA damage signalling triggers senescence-associated inflammatory cytokine secretion. Nat Cell Biol (2009) 11:973-9. doi:10.1038/ncb1909

28. Kang C, Xu Q, Martin TD, Li MZ, Demaria M, Aron L, et al. The DNA damage response induces inflammation and senescence by inhibiting autophagy of GATA4. Science (2015) 349:aaa5612. doi:10.1126/science.aaa5612

29. Young AR, Narita M, Ferreira M, Kirschner K, Sadaie M, Darot JF, et al. Autophagy mediates the mitotic senescence transition. Genes Dev (2009) 23:798-803. doi:10.1101/gad.519709

30. Knobel PA, Kotov IN, Felley-Bosco E, Stahel RA, Marti TM. Inhibition of REV3 expression induces persistent DNA damage and growth arrest in cancer cells. Neoplasia (2011) 13:961-70. doi:10.1593/neo.11828

31. Hayashi H, Higashi T, Yokoyama N, Kaida T, Sakamoto K, Fukushima Y, et al. An imbalance in TAZ and YAP expression in hepatocellular carcinoma confers cancer stem cell-like behaviors contributing to disease progression. Cancer Res (2015) 75:4985-97. doi:10.1158/0008-5472. CAN-15-0291

32. Ziegler A, Cerciello F, Bigosch C, Bausch-Fluck D, Felley-Bosco E, Ossola R, et al. Proteomic surfaceome analysis of mesothelioma. Lung Cancer (2012) 75:189-96. doi:10.1016/j.lungcan.2011.07.009
33. Consortium GT. Human genomics. The Genotype-Tissue Expression (GTEx) pilot analysis: multitissue gene regulation in humans. Science (2015) 348:648-60. doi:10.1126/science.1262110

34. Rintoul RC, Rassl DM, Gittins J, Marciniak SJ, Mesoban KC. MesobanK UK: an international mesothelioma bioresource. Thorax (2016) 71:380-2. doi:10.1136/thoraxjnl-2015-207496

35. Frei C, Opitz I, Soltermann A, Fischer B, Moura U, Rehrauer H, et al. Pleural mesothelioma side populations have a precursor phenotype. Carcinogenesis (2011) 32:1324-32. doi:10.1093/carcin/bgr127

36. Tsao MS, Wu L, Allo G, John T, Li M, Tagawa T, et al. Patient-derived xenograft establishment from human malignant pleural mesothelioma. Clin Cancer Res (2017) 23:1060-7. doi:10.1158/1078-0432.CCR-16-0844

37. van Ommen GJ, Tornwall O, Brechot C, Dagher G, Galli J, Hveem K, et al. BBMRI-ERIC as a resource for pharmaceutical and life science industries: the development of biobank-based Expert Centres. Eur J Hum Genet (2015) 23:893-900. doi:10.1038/ejhg.2014.235

38. Sun Y, Nakamura K, Wendel E, Colburn N. Progression toward tumor cell phenotype is enhanced by overexpression of a mutant p53 tumor-suppressor gene isolated from nasopharyngeal carcinoma. Proc Natl Acad Sci U S A (1993) 90:2827-31. doi:10.1073/pnas.90.7.2827

39. Morel AP, Ginestier C, Pommier RM, Cabaud O, Ruiz E, Wicinski J, et al. A stemness-related ZEB1-MSRB3 axis governs cellular pliancy and breast cancer genome stability. Nat Med (2017) 23:568-78. doi:10.1038/nm.4323

40. Szulkin A, Otvos R, Hillerdal CO, Celep A, Yousef-Fadhel E, Skribek H, et al. Characterization and drug sensitivity profiling of primary malignant mesothelioma cells from pleural effusions. BMC Cancer (2014) 14:709. doi:10.1186/1471-2407-14-709

41. Bradley JE, Ramirez G, Hagood JS. Roles and regulation of Thy-1, a context-dependent modulator of cell phenotype. Biofactors (2009) 35:258-65. doi:10.1002/biof.41

42. Cao Q, Abeysinghe H, Chow O, Xu J, Kaung H, Fong C, et al. Suppression of tumorigenicity in human ovarian carcinoma cell line SKOV-3 by microcell-mediated transfer of chromosome 11. Cancer Genet Cytogenet (2001) 129:131-7. doi:10.1016/S0165-4608(01)00442-3

43. Abeysinghe HR, Cao Q, Xu J, Pollock S, Veyberman Y, Guckert NL, et al. THY1 expression is associated with tumor suppression of human ovarian cancer. Cancer Genet Cytogenet (2003) 143:125-32. doi:10.1016/ S0165-4608(02)00855-5

44. Abeysinghe HR, Pollock SJ, Guckert NL, Veyberman Y, Keng P, Halterman M, et al. The role of the THY1 gene in human ovarian cancer suppression based on transfection studies. Cancer Genet Cytogenet (2004) 149:1-10. doi:10.1016/ S0165-4608(03)00234-6

45. Lung HL, Cheng Y, Kumaran MK, Liu ET, Murakami Y, Chan CY, et al. Fine mapping of the 11q22-23 tumor suppressive region and involvement of TSLC1 in nasopharyngeal carcinoma. Int J Cancer (2004) 112:628-35. doi:10.1002/ ijc. 20454

46. Lung HL, Bangarusamy DK, Xie D, Cheung AK, Cheng Y, Kumaran MK, et al. THY1 is a candidate tumour suppressor gene with decreased expression in metastatic nasopharyngeal carcinoma. Oncogene (2005) 24:6525-32. doi:10.1038/sj.onc. 1208812

47. Liu G, Yuan X, Zeng Z, Tunici P, Ng H, Abdulkadir IR, et al. Analysis of gene expression and chemoresistance of CD133+ cancer stem cells in glioblastoma. Mol Cancer (2006) 5:67. doi:10.1186/1476-4598-5-67

48. Yang ZF, Ho DW, Ng MN, Lau CK, Yu WC, Ngai P, et al. Significance of CD90+ cancer stem cells in human liver cancer. Cancer Cell (2008) 13:153-66. doi:10.1016/j.ccr.2008.01.013

49. Gupta PB, Onder TT, Jiang G, Tao K, Kuperwasser C, Weinberg RA, et al. Identification of selective inhibitors of cancer stem cells by high-throughput screening. Cell (2009) 138:645-59. doi:10.1016/j.cell.2009.06.034

50. Melotti A, Daga A, Marubbi D, Zunino A, Mutti L, Corte G. In vitro and in vivo characterization of highly purified human mesothelioma derived cells. BMC Cancer (2010) 10:54. doi:10.1186/1471-2407-10-54

51. Li Y, Shi X, Yang W, Lu Z, Wang P, Chen Z, et al. Transcriptome profiling of lncRNA and co-expression networks in esophageal squamous cell carcinoma by RNA sequencing. Tumour Biol (2016) 37:13091-100. doi:10.1007/ s13277-016-5227-3

52. Varley KE, Gertz J, Roberts BS, Davis NS, Bowling KM, Kirby MK, et al. Recurrent read-through fusion transcripts in breast cancer. Breast Cancer Res Treat (2014) 146:287-97. doi:10.1007/s10549-014-3019-2 
Conflict of Interest Statement: The authors declare that the research was conducted in the absence of any commercial or financial relationships that could be construed as a potential conflict of interest.

Copyright (c) 2018 Oehl, Kresoja-Rakic, Opitz, Vrugt, Weder, Stahel, Wild and Felley-Bosco. This is an open-access article distributed under the terms of the Creative Commons Attribution License (CC BY). The use, distribution or reproduction in other forums is permitted, provided the original author(s) and the copyright owner are credited and that the original publication in this journal is cited, in accordance with accepted academic practice. No use, distribution or reproduction is permitted which does not comply with these terms. 